P. Comella $\cdot$ B. Massidda $\cdot$ S. Palmeri $\cdot$ A. Farris

L. De Lucia $\cdot$ D. Natale $\cdot$ L. Maiorino $\cdot$ S. Tafuto

G. De. Cataldis $\cdot$ R. Casaretti

\title{
Biweekly oxaliplatin combined with oral capecitabine (OXXEL regimen) as first-line treatment of metastatic colorectal cancer patients: a Southern Italy Cooperative Oncology Group phase II study
}

Received: 20 December 2004 / Accepted: 8 February 2005/ Published online: 18 May 2005

(C) Springer-Verlag 2005

\begin{abstract}
Oxaliplatin $100 \mathrm{mg} / \mathrm{m}^{2}$ iv on day 1, and capecitabine $1,000 \mathrm{mg} / \mathrm{m}^{2}$ orally bid from day 1 (evening) to day 11 (morning) were administered every 2 weeks (OXXEL regimen) to 38 patients as first-line treatment for metastatic colorectal carcinoma. A total of 318 cycles were administered, with a median of 8 (range, 4-12) cycles per patient. Response rate (RR) was $45 \%(95 \%$
\end{abstract}

P. Comella $(\varangle) \cdot$ R. Casaretti

Division of Medical Oncology A, Department of Medicine,

National Tumour Institute, Via M. Semmola,

80131 Naples, Italy

E-mail: pasqualecomella@libero.it

Tel.: + 39-81-5903227

Fax: + 39-81-5903821

B. Massidda

Chair of Medical Oncology, University Medical School, Via Jenner, 09100 Cagliari, Italy

S. Palmeri

Chair of Medical Oncology, University Medical School, Piazza delle Cliniche 2, 90127 Palermo, Italy

A. Farris

Chair of Medical Oncology, University Medical School, Viale San Pietro 8, 07100 Sassari, Italy

L. D. Lucia

Unit of Medical Oncology, City Hospital,

Via Tescione 81, 81100 Caserta, Italy

D. Natale

Unit of Medical Oncology, San Massimo Hospital,

Via Battaglione degli Alpini, 65017 Penne, Italy

L. Maiorino

Unit of Medical Oncology, San Gennaro Hospital, Via San Gennaro, 80100 Naples, Italy

S. Tafuto

Division of Medical Oncology, Santa Maria Hospital,

Via Domitiana, 80078 Pozzuoli, Italy

G. D. Cataldis

Division of Medical Oncology, Da Procida Hospital, Via Calenda, 84100, Salerno, Italy confidence interval (CI), 29\%-62\%), with 7 complete responses and 10 partial responses; furthermore, 12 patients showed a stable disease, so that a disease control was achieved in $29(76 \%)$ patients. RR was greater among patients with performance status $0(52 \%)$, without weight loss $(52 \%)$, younger than 65 years $(50 \%)$, and previously unexposed to adjuvant chemotherapy $(48 \%)$, while no correlation was found with the actually delivered oxaliplatin dose intensity. Overall, haematological side effects were negligible, with no case of grade 4 toxicity, and only one patient suffering from an episode of grade 3 neutropenic fever. Severe anaemia occurred in 4 $(11 \%)$ patients, and grade 3 neuropathy affected $9(24 \%)$ patients. Median progression-free survival was $7.9(95 \%$ CI, 6.2-9.6) months, and median overall survival has not been reached yet. In conclusion, the OXXEL regimen resulted safe and active, and it deserves further evaluation in metastatic colorectal cancer patients.

Keywords Oxaliplatin Capecitabine OXXEL regimen $\cdot$ Biweekly treatment $\cdot$ Metastatic colorectal cancer

\section{Introduction}

The combination of oxaliplatin (OXA) with infusional 5-fluorouracil and leucovorin (FU/LV) (FOLFOX4 regimen) represents a new golden standard both in the adjuvant and in the palliative treatment of colon cancer. For patients with metastatic spread, FOLFOX4 was superior to IFL (irinotecan plus bolus FU/LV) in terms of response rate (RR), progression-free (PFS) and overall survival (OS), showing also a better toxicity profile [7].

French investigators have previously observed that activity of FOLFOX was related with OXA dosage and dose intensity (DI). Indeed, they reported a greater RR 
among patients treated with an OXA dosage $\geq 85 \mathrm{mg} / \mathrm{m}^{2}$ every 2 weeks than for those receiving a lower DI $(39 \%$ vs. $19 \%$ ) [11]. Increasing the OXA dosage to $130 \mathrm{mg} / \mathrm{m}^{2}$ in the FOLFOX7 biweekly regimen, a RR in $42 \%$ of patients treated in second line has been reported, associated with a median survival of 16.1 months, at a price of an acceptable toxicity [12]. However, delivery of FOLFOX regimen requires an indwelling or totally implanted central venous catheter and disposable infusional devices and/or pumps, which increase the treatment cost, may be associated with vascular complications, and cause some discomfort for the outpatient management.

Capecitabine is a parent compound of FU, which can be administered orally at a recommended dose of $1,250 \mathrm{mg} / \mathrm{m}^{2}$ bid, 12-h apart, for two consecutive weeks, and 1 week of rest. This drug has been randomly compared with standard FU/LV given i.v. monthly (Mayo Clinic regimen) either as adjuvant treatment to radical surgery and as palliative therapy. In two trials carried out in metastatic patients $[9,18]$, capecitabine obtained a higher RR $(23 \%$ vs. $15 \%$, and $27 \%$ vs. $18 \%$, respectively) with an acceptable toxicity profile: hand-foot syndrome affected on the whole $16 \%$ of patients, while severe diarrhoea occurred in $13 \%$ of them, a proportion significantly lower than that of the control arm [17].

Based on these observations, capecitabine has been tested in several phase II trials in combination with OXA (XELOX regimen) in the first and second-line treatment of metastatic patients. In the largest of these studies, 97 patients were treated in first line with OXA $130 \mathrm{mg} / \mathrm{m}^{2}$ on day 1 , and capecitabine $2,000 \mathrm{mg} / \mathrm{m}^{2}$ orally from day 1 to day 14 , recycling every 3 weeks [2]. These investigators reported a $55 \% \mathrm{RR}$, and a 19.5-month median OS, with a negligible occurrence of grade $\geq 3$ haematological and non-haematological side effects. A grade 3 neuropathy was reported in $16 \%$ of patients, while the typical hand-foot syndrome was observed in only $2 \%$ of them. In a Swiss phase II study, 26 pretreated and 43 chemonaive patients received OXA $130 \mathrm{mg} / \mathrm{m}^{2}$ on day 1 , and capecitabine $2,500 \mathrm{mg} / \mathrm{m}^{2}$ on day 1 to 14 every 3 weeks. A $49 \%$ $\mathrm{RR}$, and a median OS of 17.1 months, were reported among unpretreated patients. However, $35 \%$ of chemonaive patients, and $50 \%$ of pretreated patients, suffered from grade $\geq 3$ diarrhoea [1]. Slightly lower dosages (OXA $120 \mathrm{mg} / \mathrm{m}^{2}$ plus capecitabine 2,400 mg/ $\mathrm{m}^{2}$ for 14 days every 3 weeks) were explored in an other phase II study in first line. This combination yielded a $44 \%$ RR, and a median OS of 20 months, but severe diarrhoea affected $28 \%$ of patients, suggesting a further reduction of capecitabine dosage [19]. Recently, also a different schedule of OXA $\left(70 \mathrm{mg} / \mathrm{m}^{2}\right.$ given on days 1 and 8) has been explored in combination with capecitabine $2,000 \mathrm{mg} / \mathrm{m}^{2}$ daily for 2 of 3 weeks in a phase II randomized trial. This regimen was reported to obtain a $50.7 \%$ RR among 75 unpretreated patients [8].

With these premises in mind, we decided to assess the activity and tolerability of a combination of OXA and capecitabine given in a biweekly schedule (OXXEL regimen), trying to increase OXA DI while keeping unchanged capecitabine DI. The biweekly schedule should also allow for a more dose-dense treatment in comparison with the 3-weekly regimen.

\section{Patients and methods}

\section{Patients}

Patients were eligible for this study if they met the following inclusion criteria: histologically proven diagnosis of colorectal carcinoma; age $\geq 18$ years; Eastern Cooperative Oncology Group performance status (PS) $\leq 2$; life expectancy $\geq 3$ months; at least one bidimensionally measurable metastatic lesion; adequate bone marrow reserve (neutrophils $\geq 2.0 \times 10^{9}$ / $\mathrm{L}$, platelets $\geq 100 \times 10^{9} / \mathrm{L}$, and haemoglobin serum concentration $\geq 10 \mathrm{~g} / \mathrm{dL}$ ); normal liver function (bilirubin < 1.25xupper normal limit [UNL], ALT and AST $<2.5 \times \mathrm{UNL}$ in the absence of liver metastasis, or bilirubin $<1.25$, ALT and AST $<5 \times \mathrm{UNL}$ in the case of liver metastasis); normal renal function (serum creatinine $<1.5 \times \mathrm{UNL}$ ). Exclusion criteria were: previous chemotherapy for the metastatic disease (adjuvant treatment was permitted, provided that at least 6 months had elapsed from its discontinuation); uncontrolled metabolic disorders or active infections; inability to swallow oral medications; inflammatory bowel diseases, significant diarrhoea during the last week or bowel obstruction; previous total colectomy or ileostomy; severe cardiac arrhythmia, uncontrolled congestive cardiac failure, severe ischaemic heart disease, or acute myocardial infarction in the last 6 months; symptomatic cerebral metastasis. All patients gave an informed consent to participate in this trial, which was approved by the Independent Ethical Committee of the National Tumour Institute of $\mathrm{Na}$ ples.

\section{Initial assessment}

Medical history, physical examination, performance status, and symptoms of disease were registered at patient's entry. Blood cell count (BCC), white cell differential, biochemistry, urinalysis, and serum CEA basal value were assessed within one week from initial therapy. Chest $X$-ray and ECG were routinely performed. Target lesions were measured with computed tomography (CT) or magnetic resonance imaging (MRI) scans carried-out within 1 month before study entry.

\section{Assessment of toxicity and activity}

BCC was performed weekly, while biochemistry was repeated at the beginning of each cycle. Physical status 
and assessment of toxicity of previous cycle were checked before the start of the next cycle. Toxicity was scored according to WHO criteria [13], but a specific scale was used for OXA neurotoxicity: grade 1 was the occurrence of paraesthesia and/or dysaestesia lasting less than 1 week; grade 2 was the occurrence of paraesthesia and/or dysaestesia lasting more than 1 week; grade 3 was the occurrence of paraesthesia and/or dysaestesia persisting for more than 2 weeks; and grade 4 was neurotoxicity with pain and/or functional impairment. The worst toxicity suffered by each patient during the whole treatment was recorded.

Evaluation of activity on target lesions with CT or MRI scan was done after every four cycles. Responses were classified according to WHO criteria [13]. PFS was the time elapsed from the date of initial therapy to the date of tumour progression or death. Patients who discontinued study medication for reasons different from progression were censored for PFS analysis. OS was the time elapsed from the date of enrolment into the study to the date of death, or last follow-up.

\section{Treatment}

OXA $100 \mathrm{mg} / \mathrm{m}^{2}$ was administered diluted in $500 \mathrm{~mL}$ of $5 \%$ DW solution over $2 \mathrm{~h}$ on day 1 ; capecitabine $1,000 \mathrm{mg} / \mathrm{m}^{2}$ was assumed orally bid, $12 \mathrm{~h}$ apart, within 30 min after the end of the breakfast and evening meal, from day 1 (evening) to day 11 (morning). The total daily dose was rounded for a combination of 500 and $150 \mathrm{mg}$ tablets. Cycles were repeated every 2 weeks. Doses were unchanged throughout the whole treatment, unless a grade 3 non-haematological toxicity occurred. In this case, treatment was discontinued, and than resumed only after a complete recovery, with a $20 \%$ OXA dose reduction and a $25 \%$ capecitabine dose reduction. In the case of grade 4 non-haematological toxicity (except for alopecia), grade 4 neutropenia or thrombocytopenia lasting more than one week, or febrile neutropenia, treatment was definitely interrupted. The experimental treatment was planned for a maximum of 12 cycles. In the case of progression, second line treatment was left at the discretion of the attending physician.

\section{Statistical considerations and sample size}

RR was the main end-point of this phase II trial. We adopted a two-stage mini-max design [15]: defining as $30 \%$ the minimum RR expected for the experimental treatment, and as alternative of clinical interest a $50 \%$ $\mathrm{RR}$, at least 17 responses among a total of 39 patients were needed for accepting this hypothesis, with an alpha error $<0.05$ and an $80 \%$ power (1-beta). RR (with $95 \%$ CI) [4] was calculated on all eligible patients according to intention-to-treat analysis. Actuarial PFS and OS times were estimated using actuarial method [10].

\section{Results}

\section{Patients}

From September 6, 2003, to April, 24, 2004, 39 patients were accrued into this trial by nine centres. After registration, one patient was found to be ineligible, because of previous exposure to chemotherapy for the metastatic disease, leaving 38 patients assessable for activity and toxicity. Main characteristics of these patients are reported in Table 1 . Most patients $(71 \%)$ were males, their median age was 62 (range, 35-80) years. All patients had a fairly good PS (0 in 29 patients, 1 in 9 patients). Eleven patients had previously received adjuvant chemotherapy (FU/LV, 10 cases; FOLFIRI, 1 case). Twenty-two $(55 \%)$ patients had only one site of disease, and liver was involved in $25(66 \%)$ patients.

\section{Extent of treatment exposure}

A total of 318 cycles were administered, with a median of 8 (range, 4-12) cycles/patient. All patients received at least 4 cycles of treatment, $26(68 \%)$ patients were treated up to 8 cycles, and $8(21 \%)$ patients received a maximum of 12 cycles.

Treatment discontinuation occurred according to protocol's rules in 31 patients. Three patients refused further treatment after 7 ( 2 cases) and 8 ( 1 case) cycles, respectively, while three patients went off treatment for toxicity: one patient suffered from severe diarrhoea

Table 1 Characteristics of patients

\begin{tabular}{lll}
\hline Characteristics & No. & Percentage \\
\hline Eligible patients & 38 & 100 \\
Males & 27 & 71 \\
Females & 11 & 29 \\
Median age (range) in years, $62(35-80)$ & & \\
Performance status (ECOG) & & \\
0 & 29 & 76 \\
1 & 9 & 24 \\
Primary site & 26 & 68 \\
Colon & 12 & 32 \\
Rectum & 27 & 71 \\
Previous surgery & & \\
Previous adjuvant treatment & 10 & 26 \\
FAFU & 1 & 2 \\
FOLFIRI & 7 & 18 \\
Previous weight loss $>5 \%$ & 10 & 26 \\
Presence of symptoms & & \\
Number of disease sites & 21 & 55 \\
1 & 9 & 24 \\
2 & 8 & 21 \\
$3+$ & 25 & 66 \\
Liver & 9 & 23 \\
Lung & 9 & 23 \\
Lymph nodes & 4 & 10 \\
Peritoneal & 29 & 76 \\
CEA > 5 ng/mL & 12 & 31 \\
CEA > 100 ng/mL & & \\
\hline & & \\
\hline & &
\end{tabular}


Table 2 Activity reported among 38 patients (intent-to-treat analysis)

\begin{tabular}{lll}
\hline Response & No. & Percentage \\
\hline Complete response & 7 & 18 \\
Partial response & 10 & 26 \\
No change & 12 & 32 \\
Progressive disease & 8 & 21 \\
Not assessed & 1 & 3 \\
Total patients & 38 & 100 \\
Overall response rate $(95 \% \mathrm{CI})$ & $45 \%$ & \\
& $(29-62 \%)$ & \\
\hline
\end{tabular}

requiring hospitalization and forced rehydration, but he eventually recovered from this toxicity; another patient discontinued her treatment after four cycles for a persistent grade 3 liver toxicity (increase of serum ASAT and ALAT); and a third patient was withdrawn for persistent neurosensory toxicity after 10 cycles (OXA cumulative dose, $949 \mathrm{mg} / \mathrm{m}^{2}$ ). A last patient, after achieving a substantial shrinkage of hepatic deposits after 8 cycles, underwent radical liver surgery.

Median dose intensity (DI) over the first 4 cycles was 46 (range, 31-56) $\mathrm{mg} / \mathrm{m}^{2} /$ week for OXA, and 9.2 (range, $1.0-10.8) \mathrm{g} / \mathrm{m}^{2} /$ week for capecitabine. Corresponding DI over 8 cycles were $42 \mathrm{mg} / \mathrm{m}^{2} /$ week (range, 29-50) and 8.9 (range, 3.5-10.7) respectively. Mean cumulative dosage of OXA was 832 (range, 383-1,200) $\mathrm{mg} / \mathrm{m}^{2}$, while mean cumulative dosage of capecitabine was 162 (range, $8.0-250) \mathrm{g} / \mathrm{m}^{2}$.

\section{Activity}

A complete response (CR) was achieved in 7 patients, and a partial response (PR) in 10 patients, giving an RR of $45 \%(95 \%$ CI, 29\%-62\%) Responses were observed after a median of 2.3 (range, 1.8-7.2) months, and 11 of $17(69 \%)$ patients achieved such result within 4 months from initial therapy. In all but one patient, who underwent surgical liver resection earlier, responses were confirmed 2 months after their first assessment. Median duration of responses was 5.5 months. Furthermore, 12 patients were classified in stable disease. On the whole, 29 (76\%) patients achieved at least a control of tumour growth (response or stabilization) with the treatment on study. Moreover, we would report that 8 of $12(66 \%)$ patients with a basal CEA value $\geq 100 \mathrm{ng} / \mathrm{mL}$ showed a $>50 \%$ drop of this value during treatment.

Analysis of treatment effect showed that RR was higher in patients with PS 0 as opposed to PS $1(52 \%$ vs. $22 \%$ ), for patients without previous weight loss $(52 \%$ vs. $14 \%)$, for patients younger than 65 years $(50 \%$ vs. $33 \%)$, and for those unexposed to adjuvant chemotherapy $(48 \%$ vs. $36 \%)$. On the contrary, activity was not substantial different among patients with only one disease site (48\%) and those with two or more sites $(41 \%)$. Noteworthy, lung metastases also showed a high response rate with this treatment $(44 \%)$.

Despite the fact that the regimen was intended as a more dose-dense treatment, and it was devised with the aim of intensifying the OXA delivery, no apparent correlation between RR and OXA DI was seen. Indeed, RR was $40 \%$ among patients receiving an $\mathrm{OXA} \mathrm{DI}_{4}$ of at least $46 \mathrm{mg} / \mathrm{m}^{2} /$ week (which was the median actually delivered DI over the first 4 cycles) in comparison with $44 \%$ among those receiving a lower $\mathrm{DI}_{4}$.

\section{Toxicity}

Worst acute toxicity registered for each patient is reported in Table 3. Overall, haematological side effects were negligible, with no case of grade 4 toxicity, and only a patient suffering from an episode of grade 3 neutropenic fever. Some decrease in haemoglobin serum level occurred during treatment in $15(39 \%)$ patients, but it was of grade 3 in only 4 of them (11\%). As for non-haematological side effects, gastrointestinal toxicity was quite mild: only $5(13 \%)$ patients suffered from severe diarrhoea, which required hospitalisation in one case. Moreover, we would underline that the typical hand-foot syndrome caused by capecitabine was very rare and mild. This finding might be explained by the shorter exposure to capecitabine with our regimen in comparison with the 2-week-on and 1-week-off schedule. Thirty $(79 \%)$ patients complained of some neurotoxicity, which was of grade $\geq 3$ in $9(24 \%)$ of them. As mentioned, this toxicity forced OXA discontinuation in one case. Severe peripheral neuropathy was clearly dosedependent, because it occurred in 7 of $23(30 \%)$ patients treated with an OXA cumulative dosage $\geq 800 \mathrm{mg} / \mathrm{m}^{2}$, as opposed to only 2 of $15(13 \%)$ patients receiving a lower cumulative dosage.

\section{Follow-up}

As of October 30, 2004, with a median follow-up of 10 (range, 6-13) months, $19(50 \%)$ patients have shown

Table 3 Acute toxicity by patients $(n=38)$

\begin{tabular}{llllll}
\hline Toxicity & \multicolumn{3}{l}{$\begin{array}{l}\text { WHO grade (number } \\
\text { of patients) }\end{array}$} & $3+4(\%)$ \\
\cline { 2 - 4 } & 1 & 2 & 3 & 4 & \\
\hline Neutropenia & 9 & 3 & 1 & 0 & 3 \\
Febrile neutropenia & - & - & 1 & 0 & 3 \\
Thrombocytopenia & 6 & 4 & 3 & 0 & 8 \\
Anaemia & 6 & 5 & 4 & 0 & 11 \\
Vomiting & 13 & 8 & 0 & 0 & 0 \\
Diarrhoea & 9 & 5 & 4 & 1 & 13 \\
Stomatitis & 4 & 1 & 0 & 0 & 0 \\
Hair loss & 1 & 5 & 0 & 0 & 0 \\
Skin & 3 & 2 & 0 & 0 & 0 \\
Liver & 8 & 3 & 1 & 0 & 3 \\
Neuropathy (Lévi scale) & 12 & 9 & 8 & 1 & 24 \\
\hline
\end{tabular}


tumour progression; therefore, the estimated median PFS time was 7.9 (95\% CI, 6.2-9.6) months. As salvage treatment, nine patients received irinotecan-based chemotherapy, and one patient underwent a local radiotherapy. At the time of this analysis, only two patients had died because of their cancer; therefore, the median OS has not been reached yet.

\section{Discussion}

This study aimed at assessing the tolerability and activity of a combination of OXA and capecitabine recycled every 2 weeks in patients with metastatic colorectal carcinoma. Results of this trial demonstrated the efficacy of this treatment, which yielded a major response in $45 \%$ of patients. We recognize that most of our patients showed favourable baseline characteristics, which were associated with a higher RR. However, we would underline the activity reported by this treatment also in patients with more than one site of disease $(41 \%)$. Moreover, we believe it is worth noting the activity we have seen in lung metastases $(44 \%)$, which are usually less sensitive to cytotoxic treatment.

This activity was obtained with acceptable occurrence of side effects: haematological toxicity was negligible, and diarrhoea and hand-foot syndrome were usually mild. These results might be explained by the shorter exposure to capecitabine in our biweekly regimen (10 days) as compared with the 3-weekly schedule (14 days). Indeed, a strategy of increasing the dose intensity of capecitabine has been pursued in a previous phase II randomized trial: OXA $85 \mathrm{mg} / \mathrm{m}^{2}$ was given on day 1 , while capecitabine $3,500 \mathrm{mg} / \mathrm{m}^{2}$ was administered daily for 7 days, recycling every 2 weeks. This biweekly regimen was able to deliver a $34 \%$ higher dose intensity of capecitabine in comparison with the 3-weekly administration of OXA $130 \mathrm{mg} / \mathrm{m}^{2}$ on day 1 , and capecitabine $2,000 \mathrm{mg} / \mathrm{m}^{2}$ daily for 14 days. Interestingly, occurrence and severity of toxicity were similar with the two regimens. This good tolerability has been explained by the longer overall "drug holidays" with the biweekly dose-intense capecitabine regimen as opposed to the 3-weekly regimen. Moreover, a greater activity (higher RR, longer PFS) was reported with the biweekly regimen [14].

In our trial, we have noted that peripheral neuropathy affected most of our patients, and grade $\geq 3$ occurred in $24 \%$ of them. Also in our experience, neurotoxicity was related with the cumulative dosage of OXA. Since only responder patients received the study medication for as long as for 12 cycles, we may infer that peripheral neuropathy could be considered as the price to be paid for obtaining a clinical benefit from this treatment. On the other hand, most responses were seen in our series within 4 months of treatment; therefore, a possible way to circumvent the occurrence of this side effect could be planning a dose reduction (or even discontinuation) of OXA after an intensive administration over 6-8 cycles, and its eventual reintroduction thereafter. Such a policy of OXA stop and go has been assessed in a randomized study (OPTIMOX trial) comparing the FOLFOX7 regimen (which include OXA $130 \mathrm{mg} / \mathrm{m}^{2}$ in each cycle) with the standard FOLFOX4 regimen: in this trial, severe neurotoxicity occurred in $13 \%$ of patients in the experimental arm as opposed to $19 \%$ of the control arm [5].

However, putting our results in the context of other experiences with this combination, it could be argued that the OXXEL regimen yielded a RR and a PFS similar to those obtained with the 3-weekly schedule. Indeed, RRs ranging from $44 \%$ to $55 \%$, and median PFS times included between 5.9 months and 8.2 months, have been reported with the XELOX regimen $[1,2,19]$. Moreover, we have not seen in our trial a correlation between activity and OXA DI that could justify the biweekly recycling. However, the number of patients was small, and a dose-effect relationship cannot be ruled out by our findings.

On the other hand, we would underline that severe diarrhoea and skin toxicity were quite uncommon with the OXXEL as compared with their reported frequencies with the XELOX regimen. Of course, a positive impact on patient's quality of life could support our choice. The present pilot trial was not designed to give an answer to this hypothesis; therefore, we have set up the SICOG 0401 randomized trial, which is currently comparing in terms of efficacy, toxicity and quality of life the OXXEL regimen with our OXAFAFU reference treatment [3].

Other ongoing phase III trials are also comparing the XELOX and FOLFOX4 regimens, with or without the addition of bevacizumab, either in the adjuvant and in the palliative setting. Results of these studies will elucidate whether capecitabine could definitely replace FU/LV in combination with OXA. Moreover, exciting results have recently been reported combining inhibitors of the epidermal growth factor receptor (EGFR) pathway with the FOLFOX4 regimen in metastatic patients. Indeed, the addition of weekly cetuximab to the FOLFOX4 has been reported to obtain an objective response in $70 \%$ of patients selected on the basis of the positive EGFR expression on tumour [16]. Moreover, the FOLFOX4 regimen combined with oral gefitinib showed activity in $78 \%$ of patients previously unexposed to chemotherapy for their metastatic disease, and in $36 \%$ of previously treated patients [6]. On the basis of our preliminary safety and activity data, we believe that also the OXXEL regimen could serve as a backbone for assessing the combination of cytotoxic and targeted biologic treatments in metastatic colorectal cancer patients.

\section{References}

1. Borner MM, Dietrich D, Stupp R et al (2002) Phase II study of capecitabine and oxaliplatin in first- and second-line treatment of advanced or metastatic colorectal cancer. J Clin Oncol 20:1759-1766 
2. Cassidy J, Tabernero J, Twelves C, et al (2004) XELOX (capecitabine plus oxaliplatin): active first-line therapy for patients with metastatic colorectal cancer. J Clin Onc 22:2084 2091

3. Comella P, Massidda B, Filippelli G, et al (2004) Oxaliplatin (OXA) plus levo-folinic acid (l-FA) and 5-fluorouacil (FU) i.v. bolus (OXAFAFU) is more active than irinotecan (IRI) plus levo-folinic acid (l-FA) and 5-fluorouacil (FU) i.v. bolus (IRIFAFU) in advanced colorectal carcinoma (ACC): Final results of the Southern Italy Cooperative Oncology Group (SICOG) trial 0103. Ann Oncol 15 (Supplement 3): iii72, abstract\# 271PD

4. Cox DR (1970) The analysis of binary data. Methnem, London

5. De Gramont A, Cervantes A, André T et al (2004) OPTIMOX study: FOLFOX7/LV5FU2 compared to FOLFOX4 in patients with advanced colorectal cancer. Proc Am Soc Clin Oncol 251s, abstract\# 3525

6. Fisher GA, Kuo T, Cho CD et al (2004) A phase II study of Gefitinib in combination with FOLFOX-4 (IFOX) in patients with metastatic colorectal cancer. Proc Am Soc Clin Oncol 248s, abstract\# 3514

7. Goldberg RM, Sargent DJ, Morton RF et al (2004) A randomized controlled trial of fluorouracil plus leucovorin, irinotecan, and oxaliplatin combinations in patients with previously untreated metastatic colorectal cancer. J Clin Oncol 22:23-30

8. Grothey A, Jordan K, Kellner O et al (2003) Capecitabine plus irinotecan (CAPIRI) vs capecitabine plus oxaliplatin (CAPOX) as first-line therapy of advanced colorectal cancer (ACRC): updated results of a randomized phase II trial. Eur J Cancer S90, abstract\# 295

9. Hoff PM, Ansari R. Batisti G et al (2001) Comparison of oral capecitabine versus intravenous fluorouracil plus leucovorin as first-line treatment in 605 patients with metastatic colorectal cancer: results of a randomized phase III study. J Clin Oncol 19:2282-2292

10. Kaplan EL, Meier P (1958) Non-parametric estimation from incomplete observations. J Am Stat Assoc 53:457-481
11. Maindrault-Gœbel F, de Gramont A, Louvet C et al (2000) Evaluation of oxaliplatin dose intensity in bimonthly leucovorin and 48-hour 5-fluorouracil continuous infusion regimens (FOLFOX) in pretreated metastatic colorectal cancer. Oncology Multidisciplinary Research Group (GERCOR). Ann Oncol 11:1477-1483

12. Maindrault-Gœbel F, de Gramont A, Louvet C et al (2001) High-dose intensity oxaliplatin added to the simplified bimonthly leucovorin and 5-fluorouracil regimen as second-line therapy for metastatic colorectal cancer (FOLFOX 7). Eur J Cancer 37:1000-1005

13. Miller AB, Hoogstraten B, Staquet M, Winkler V (1981) Reporting results of cancer treatment. Cancer 47:207-214

14. Scheithauer WS, Kornek GV, Raderer M, et al (2003) Randomized multicenter phase II trial of two different schedules of capecitabine plus oxaliplatin as first-line treatment in advanced colorectal cancer. J Clin Oncol 21:1307-1312

15. Simon R (1989) Optimal two stage design for phase II clinical trials. Contr Clin Trial 10:1-10

16. Tabernero JM, Van Cutsem E, Sastre J et al (2004) An international phase II study of cetuximab in combination with oxaliplatin/5-fluorouracil (5-FU)/folinic acid (FA) (FOLOFOX4) in the first-line treatment of patients with metastatic colorectal cancer (CRC) expressing Epidermal Growth Factor Receptor (EGFR): Preliminary results. Proc Am Soc Clin Oncol 248s, abstract\# 3512

17. Twelves C, on behalf of the Xeloda Colorectal Cancer Group (2002) Capecitabine as first-line treatment in colorectal cancer: pooled data from two large, phase III trials. Eur J Cancer 38:S15-S20

18. Van Cutsem E, Twelves C, Cassidy J et al (2001) Oral capecitabine compared with intravenous 5-fluorouracil plus leucovorin (Mayo Clinic regimen) in patients with metastatic colorectal cancer: results of a large phase III study. J Clin Oncol 19:4097-4106

19. Zeuli M, Nardoni C, Pino MS et al (2003) Phase II study of capecitabine and oxaliplatin as first-line treatment in advanced colorectal cancer. Ann Oncol 14:1378-1382 